\title{
A LEAST SQUARES APPROACH FOR THE RECONSTRUCTION OF NERVE FIBER ORIENTATIONS FROM TILTABLE SPECIMEN EXPERIMENTS IN 3D-PLI
}

\author{
Daniel Schmitz ${ }^{1}$, Katrin Amunts ${ }^{1}$, Thomas Lippert ${ }^{2}$, Markus Axer $^{1}$ \\ ${ }^{1}$ Institute of Neuroscience and Medicine (INM-1), Research Center Jülich \\ ${ }^{2}$ Jülich Supercomputing Center, Research Center Jülich
}

\begin{abstract}
3D-Polarized Light Imaging has become a unique technique to study the fiber architecture of unstained brain sections at the meso- and microscale. It exploits the intrinsic birefringence of nerve fibers which is measured with a customized polarimeter in which the brain section is placed on a tiltable specimen stage. So far, a computationally fast analytical method based on the discrete Fourier transformation to analyze the data acquired with the tiltable specimen stage has been used. In this study, we propose a new algorithm based on a fitting approach which provides an improved stability against measurement noise resulting in a more realistic orientation interpretation, in particular for low signals.

For the first time, it is demonstrated how fiber courses at the boundary of white and grey matter can robustly be reconstructed with 3D-PLI. This significantly improves the reliability of mapping the cortex based on 3D-PLI data.
\end{abstract}

Index Terms - Neuroimaging, Fiber Architecture, Modelling

\section{INTRODUCTION}

The brain's nerve fiber architecture is a highly complicated structure consisting of billions of interconnected neurons. Knowledge about the fiber pathways is key to the understanding of the human connectome. 3D-PLI has established its role bridging between macro- and microscale with unique abilities for neuroanatomical studies [1,2]. It enables the ex-vivo measurement of unstained histological brain sections in a customized polarimetric setup revealing fiber tracts at the $\mu \mathrm{m}$ scale. The physical principle behind 3D-PLI is the birefringence of myelinated fibers which causes a change of the polarization of incoming light depending on the spatial orientation and density of fibers.

While the reconstruction of the in-plane orientation of the nerve fiber is straight forward, the information about the outof-plane orientation is entangled with the local fiber density. Until now, one analytical approach has been presented to disentangle both parameters from each other [3] which, however suffers from noise instability.
We aim for an optimization based scheme to reconstruct fiber orientation and fiber density independently from each other to ensure a more accurate reconstruction. Our contribution is the derivation and implementation of the least squares solver. It is then evaluated on simulated and experimental data and compared with the analytical approach.

\section{3D-PLI}

The optical setup depicted in Fig. 1 consists of an LED light source emitting unpolarized light which then passes a linear polarizer, a quarter-wave retarder, a brain section mounted on a tiltable specimen stage, a second linear polarizer serving as an analyzer and a camera which acquires images at a pixel size of $64 \times 64 \mu \mathrm{m}^{2}$ [2]. The tilting stage enables rotations around the $\mathrm{x}$ - and $\mathrm{y}$-axis of up to $\tau= \pm 8^{\circ}$. The polarizers and the retarder are rotated simultaneously in steps of $10^{\circ}$ from $0^{\circ}$ to $170^{\circ}$. At each position an image is acquired.

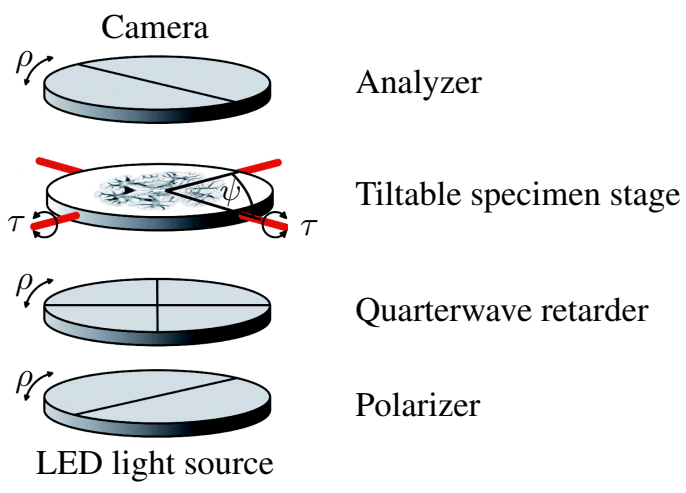

Fig. 1: Experimental setup [3]

A nerve fiber is modeled as an uniaxial birefringent crystal whose principal axis is oriented along the fiber orientation. According to this model the measured light intensity in each pixel yields a sinusoidal profile depending on the rotation angle $\rho$ by

$$
I(\rho, \varphi, \alpha, t)=I_{T}\left(1+\sin (2(\rho-\varphi)) \sin \left(\frac{\pi}{2} t \cos (\alpha)^{2}\right)\right)
$$

with the transmitted light intensity $I_{T}$ (transmittance), the inplane fiber orientation $\varphi$ (direction angle), out-of-plane fiber 
orientation $\alpha$ (inclination angle) and the fiber density $t$ (for readability purposes we denote $t_{r e l}$ as $t$ and $I_{T}$ as $\frac{I_{0}}{2}$ in contrast to standard 3D-PLI convention) [2]. The 3D-PLI coordinate system is depicted in Fig. 2: the range of the orientation angles is $\varphi \in[0, \pi]$ and $\alpha \in\left[-\frac{\pi}{2}, \frac{\pi}{2}\right]$. The fiber density parameter $t$ is given by $t=4 t_{s} \Delta n / \lambda$ taking into account the section thickness $t_{s}$, birefringence $\Delta n$ and the illumination wavelength $\lambda$ [2].

The established analysis of this profile is a Fourier analysis resulting in the transmittance $I_{T}$ as the average transmitted light intensity, the direction angle $\varphi$ as the phase of the sine curve and the relative amplitude of the sine $\left|\sin \left(\frac{\pi}{2} t \cos (\alpha)^{2}\right)\right| \quad$ (retardation).

As $t$ and $\alpha$ are mapped onto one value, it is impossible to reconstruct them independently from each other without further measurement information. This is accomplished by means of the tilting stage: by applying a welldefined rotation to the specimen, the

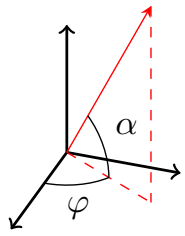

Fig. 2: 3D-PLI coordinate system intensity profile also changes in a predefined manner. For every tilting position (in routine both axes tilt by $8^{\circ}$ and $-8^{\circ}$ so the tilting positions $\psi=0^{\circ}, 90^{\circ}, 180^{\circ}, 270^{\circ}$ are utilized) an image series is acquired. All images taken with a tilted stage are registered onto the flat measurement using a projective linear transformation.

So far, an analytical approach based on a discrete Fourier transform of the retardation data (DFT algorithm) has been presented to extract $t$ and $\alpha$ independently from each other [3]. As very steep fibers $\left(|\alpha|>80^{\circ}\right)$ and slightly myelinated fibers $(t<0.1)$ cause very low retardation signals, the change between the retardation value at the different tilting positions might be challenging to interpret due to measurement noise if being at the same order of magnitude as the native birefringent signal. Therefore the computationally fast DFT algorithm comes with the drawback of noise instability for these two cases.

We seek to improve the reconstruction of $\alpha$ and $t$ by not only exploiting the amplitude of the sine curves, but the whole sinusoidal profiles. Furthermore, the analytical approach is replaced by a more robust fitting procedure.

\section{LEAST SQUARES SOLVER}

We denote the tilting position with the index $j$ and the rotation angle with index $i$. For every tilting position $j$ the modelled fiber orientation given by $\vec{r}_{j}$ can be calculated by applying the appropriate rotation on the true orientation vector $\vec{r}$ as $\vec{r}_{j}=R_{j} \vec{r}$ (rotation matrix $R_{j}$ ) yielding tilted direction angles $\varphi_{j}$ and tilted inclination angles $\alpha_{j}$. Due to refraction at the brain tissue, the actual angle of the tilted light path through the tissue is reduced to $\tau_{\text {int }} \approx 5.7^{\circ}$ for a tilt of the tilting stage by $\tau=8^{\circ}$. As a consequence of the longer light path through the tissue the fiber density $t$ has to be adjusted to $t_{j}=t / \cos \left(\tau_{\text {int }}\right)$ [3]. The transition from the planar measurement with the fiber parameters $(\varphi, \alpha, t)$ to the tilted measurements with the tilted fiber parameters $\left(\varphi_{j}, \alpha_{j}, t_{j}\right)$ results in a slightly different intensity curve $I_{j}$ for every tilting position.

As the transmittance $I_{j, T}$ is effected by additional refraction and absorptions effects in a tilted measurement, we do not include it in the fitting process. To eliminate its effects on the evaluation of the fiber orientation we define the normalized light intensity $I_{N_{j i}}=\frac{I_{j i}}{I_{j, T}}-1$ which is limited to the range $[-1,1]$. For a measurement of $N_{P}$ rotation angles error propagation results in the following standard deviation $\sigma_{N_{j i}}$ of $I_{N_{j i}}$ :

$$
\sigma_{N_{j i}}=\left[\frac{g I_{j i}}{I_{j, T}^{2}}+\frac{g I_{j i}^{2}}{N_{P} I_{j, T}^{3}}\right]^{\frac{1}{2}}
$$

with the gain factor $g$ accounting for the level of measurement noise $I \sim \mathcal{N}(I, \sqrt{g \cdot I})$. In the current setup the gain factor was experimentally determined to be $g=3$ [4].

According to the 3D-PLI model the normalized light intensity $f_{j i}$ is also given by $\frac{I_{j i}}{I_{j, T}}-1$ yielding

$$
f_{j i}\left(\varphi_{j}, \alpha_{j}, d_{j}, \rho_{i}\right)=\sin \left(2\left(\rho_{i}-\varphi_{j}\right)\right) \sin \left(\frac{\pi}{2} t_{j} \cos \left(\alpha_{j}\right)^{2}\right)
$$

Similar to minimizing the weighted sum of the squared residuals between one function and data points as in a common weighted least squares approach, we now seek to minimize the sum of the squared residuals between $f_{j i}$ and the normalized intensities $I_{N_{j i}}$ of all tilting positions $N_{T}$ and rotation angles $N_{P}$ weighted by the inverse standard deviation $w_{j i}=\sigma_{N_{j i}}^{-1}$. The optimization problem can be formulated as minimizing the following objective function:

$$
\chi^{2}=\sum_{j=0}^{N_{T}} \sum_{i=0}^{N_{P}}\left(\left(f_{j i}\left(\varphi_{j}, \alpha_{j}, t_{j}, \rho_{i}\right)-I_{N_{j i}}\right) \cdot w_{j i}\right)^{2}
$$

subject to $\varphi \in[0, \pi], \alpha \in\left[-\frac{\pi}{2}, \frac{\pi}{2}\right], t \geq 0$. Two problems have to be solved to find the global minimum: the boundaries of the parameters and a suitable starting point for the optimizer.

As the direction angle $\varphi_{0}$ can be directly derived from the planar measurement, it provides a good starting point for $\varphi$. The first guesses for $\alpha$ and $t$ are determined by brute force minimization of a $6 \times 6$ grid $\left(\left[\varphi_{0}, \alpha_{l}, t_{k}\right], k, l=1, \ldots, 6\right)$ equidistantly spanning the parameter space.

Least-squares solvers capable of dealing with hard boundaries are available but in our case a more convenient solution is to allow all values for the parameters and exploit the symmetry of the problem [4]. As $f(\varphi, \alpha, t)=-f(\varphi, \alpha,-t)$, it is sufficient to take the absolute value of $t=|t|$ in each optimization iteration. Considering the symmetry of spherical coordinates, the unbounded orientation given as $\left(\varphi_{u}, \alpha_{u}\right)$ can be transformed back into the standard 3D-PLI parameter 


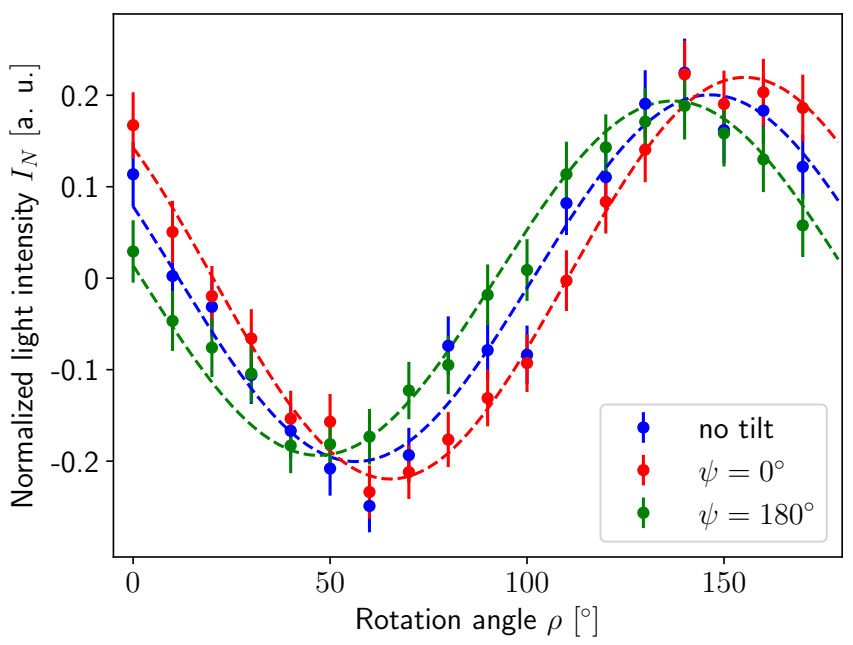

Fig. 3: Example of the fitting procedure. For illustration issues only two tilting positions are depicted. Fit result: $\varphi=101^{\circ}, \alpha=-60^{\circ}, t=0.5 . R^{2}=0.97$

space by the following transformations:

$\alpha=\left(\left(\alpha_{u}+\frac{\pi}{2}\right) \bmod \pi-\frac{\pi}{2}\right) \operatorname{sign}\left(-\left\lfloor\frac{\varphi_{u}}{\pi} \bmod 2\right\rfloor+\frac{1}{2}\right)$

$\varphi=\varphi_{u} \bmod \pi$

These transformations have to be applied before calculating $f$ in each optimization iteration.

With the starting point given by the brute force minimization and the mentioned transformations the LevenbergMarquardt algorithm as implemented in scipy [5] is used to find the global minimum of the objective function. To reduce the enormous computation time (about 4 core hours for one human brain section), the algorithm was parallelized pixelwise and ported to the JURECA system [6]. This newly developed algorithm is denoted as Robust Orientation Fitting via Least Squares algorithm (ROFL algorithm). One example of the fit result for experimental data of one pixel of a human hemisphere is depicted in Fig. 3: the normalized intensity curves for the planar measurement and for tilts by $\tau=8^{\circ}$ into the directions $\psi=0^{\circ}$ and $\psi=180^{\circ}$ are plotted along with the fitted intensity curves according to the model.

\section{RESULTS}

\subsection{Simulated data}

In order to test the numerical stability against measurement noise, simulations were carried out. As the inclination angle is more challenging to reconstruct, the direction angle was fixed at $\varphi=30^{\circ}$. Regarding the inclination angle, only positive inclinations are simulated as the inclination sign does not effect the reconstruction accuracy. Similar to [3], synthetic data is generated by specifying a ground truth orientation vector, rotating it into the 4 tilting directions of the routine measurement and then computing sinus curves for each tilting po-

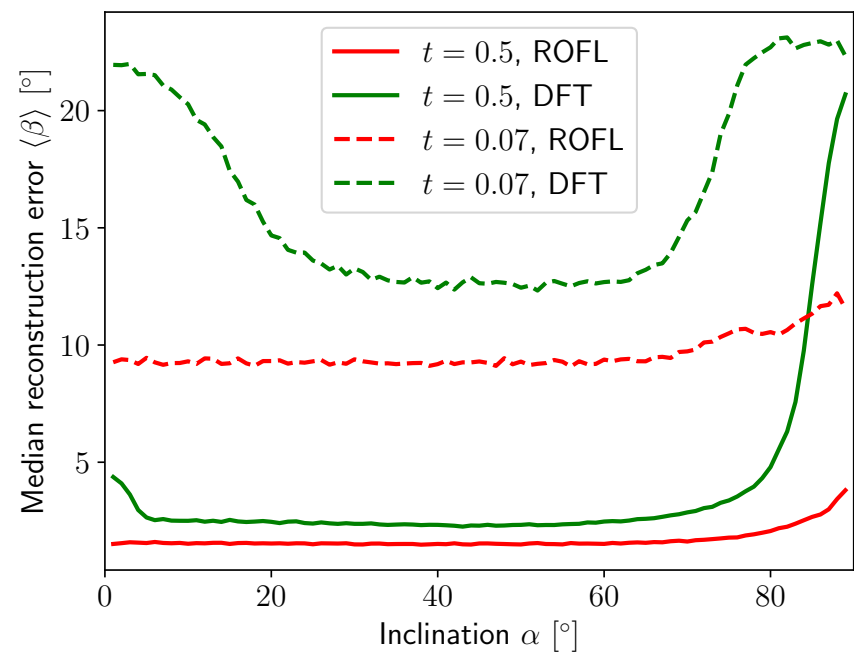

Fig. 4: Simulation results. The median reconstruction error $\langle\beta\rangle$ of the ROFL algorithm (red line) and the DFT algorithm (green line) is plotted against the fiber inclination angle $\alpha$ for $t=0.5$ and $t=0.07$ (dashed line)

sition according to the 3D-PLI model for a light intensity $I_{T}$ of 2500 (a typical intensity for human brain sections) for two fiber densities: $t=0.07$ representing a fiber at the white/gray matter boundary and $t=0.5$ representing a white matter scenario. For both densities fiber orientations with inclinations $\alpha$ from $1^{\circ}, 2^{\circ}, \ldots, 89^{\circ}$ were simulated as ground truth orientation. Each light intensity was distributed according to the distribution of the current polarimetric setup: $I \sim \mathcal{N}(I, \sqrt{3 I})$. 10.000 samples of sinusoidal profiles each were generated to provide a sufficient statistic.

These sinus profiles were then analyzed with the ROFL and the DFT algorithm resulting in reconstructed fiber orientations. The reconstruction error was assessed by calculating the acute angle $\beta$ between the simulated ground truth orientation and the orientations calculated by the ROFL and the DFT algorithm. For each combination of $t$ and $\alpha$ the overall reconstruction accuracy $\langle\beta\rangle$ is then given by the median error of all 10.000 samples. The results are plotted in Fig. 4: the ROFL algorithm provides a smaller reconstruction error in both cases, especially for very flat and very steep fibers. For very steep fibers, this can be explained by the very low retardation values corrupted by measurement noise. For in-plane fibers with $\alpha \approx 0^{\circ}$, the increased error of the DFT algorithm arises as the retardation gradient with respect to the different tilting positions yields 0 in this case: $\left.\frac{\partial \sin \delta}{\partial \alpha}\right|_{\alpha=0^{\circ}}=0$.

\subsection{Experimental data}

The ROFL and DFT algorithms were applied to a series of 236 consecutive coronal sections of a right human hemisphere (section thickness: $70 \mu \mathrm{m}$ ) which were measured with the standard measurement protocol of 4 tilting positions. All sections underwent a registration process to regain a coherent 3D-volume. The fiber orientations were reoriented based on 

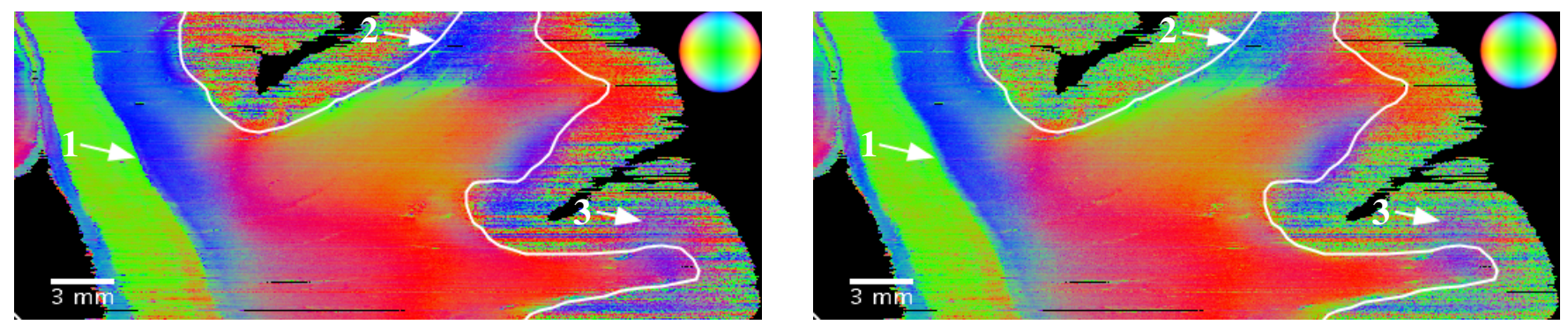

Fig. 5: Experimental results. Fiber orientation maps of a stack of 236 registered consecutive coronal sections of a human hemisphere processed with the ROFL algorithm (left) and the DFT algorithm (right). White line: manual delineation of the white/gray matter boundary based on the corresponding light transmittance map. Arrows highlight differences between both maps. Note that line effects in grey matter originate from the registration process and misinterpretations in grey matter

the spatial transformations obtained by the 3D-reconstruction scheme. The stack of all sections was virtually resliced into the plane perpendicular to the sectioning plane to highlight if the obtained orientations are coherent over the whole volume. One view of this resliced volume is shown in Fig. 5 enabling a comparison between the both algorithms based on the color coded fiber orientations. The depicted boundary between white and gray matter was delineated manually.

Both algorithms result in very similar orientations. Minor differences occur at boundaries between different anatomical regions (arrow 1) and at the boundary between white and gray matter, where the ROFL algorithm seems to estimate a smoother and less noisy fiber orientation map than the DFT algorithm (arrows 2/3). The average mean squared error between the measured and predicted light intensities of all pixels is $15 \%$ lower for the results of the ROFL algorithm than for the results of the DFT algorithm.

\section{CONCLUSION}

We presented a new algorithm based on a weighted least squares approach for the analysis of 3D-PLI data which provides a higher reconstruction accuracy on simulated data than the analytical approach. For the first time, it was demonstrated how very steep fibers can reliably reconstructed for high fiber densities in simulated data.

For experimental data, the results of the new approach are mostly consistent with the analytical approach and might offer deeper insights into gray matter regions. The lower mean squared error also indicates a better reconstruction of the underlying fiber architecture.

While the simulation results indicate that greater differences could be expected for fibers oriented perpendicular to the sectioning plane, no major differences were found in the experimental data. The explanation for this behavior might be the partial volume effect: at a voxel size of $64 \times 64 \times 70 \mu \mathrm{m}^{3}$ many voxels cannot be fully described by only one fiber orientation. As in-plane fibers cause a much higher amplitude in the measured sinusoidal signal than steep fibers, they are also preferably reconstructed by the ROFL algorithm in case of a fiber crossing. As a consequence 3D-PLI is inherently biased towards in-plane fibers, which needs to be adressed in future studies.

Acknowledgements: We express our gratitude to Sascha Münzing and Martin Schober for the 3D registration of the experimental data. The brain tissue was obtained from the Brain Bank Center Munich in accordance with the written informed consent of the subject and with legal and ethical requirements. This project has received funding from the European Union's Horizon 2020 Research and Innovation Programme under Grant Agreement No. 7202070 (HBP SGA1). We would gratefully acknowledge the computing time granted by the John von Neumann Institute for Computing (NIC) and provided on the supercomputer JURECA (Jülich-Supercomputing-Centre, 2016) at Jülich Supercomputing Centre (JSC).

\section{REFERENCES}

[1] M. Zeineh et al., "Direct visualization and mapping of the spatial course of fiber tracts at microscopic resolution in the human hippocampus," Cerebral Cortex, vol. 27, 2016.

[2] M. Axer et al., "High-resolution fiber tract reconstruction in the human brain by means of three-dimensional polarized light imaging," Frontiers in neuroinformatics, vol. 5, pp. 34, 122011.

[3] H. Wiese et al., "Polarized light imaging of the human brain: a new approach to the data analysis of tilted sections," in Proc. SPIE, 2014, vol. 9099.

[4] H. Wiese, Enhancing the Signal Interpretation and Microscopical Hardware Concept of 3D Polarized Light Imaging, Ph.D. thesis, Bergische Universität Wuppertal, 2017.

[5] E. Jones et al, "SciPy: Open source scientific tools for Python," 2001-.

[6] Jülich Supercomputing Centre, "JURECA: Generalpurpose supercomputer at Jülich Supercomputing Centre," Journal of large-scale research facilities, vol. 2, no. A62, 2016. 